\title{
Lumbar spinal canal stenosis: An early sign of amyloid transthyretin related amyloidosis
}

\author{
Alessandro Graziani ${ }^{1}$, Patrizia Cenni ${ }^{2}$, Matteo Lisi $^{3}$, Marco Domenicali ${ }^{4}$, Ludovico Graziani ${ }^{5}$ \\ 'Department of Internal Medicine, Santa Maria Delle Croci, Ravenna, Italy \\ 2Department of Radiology, Santa Maria Delle Croci, Ravenna, Italy \\ 3Department of Cardiology, Santa Maria Delle Croci, Ravenna, Italy \\ ${ }^{4}$ Department of Internal Medicine, University of Bologna, Bologna, Italy \\ ${ }^{5}$ Department of Genetic Medicine, Bambino Gesù Children's Hospital, Roma, Italy
}

\author{
Correspondence to: \\ Alessandro Graziani, MD, PhD, \\ Department of Internal Medicine, \\ Santa Maria Delle Croci, \\ Viale Randi 23, Ravenna, Italy, \\ phone: + 390544285322 ; \\ e-mail: alessandro.graziani@ \\ auslromagna.it \\ Copyright by the Author(s), 2022 \\ DOI: 10.33963/KP.a2021.0179 \\ Received: \\ November 11, 2021 \\ Accepted: \\ December 13, 2021 \\ Early publication date: \\ December 13, 2021
}

Amyloid transthyretin-related amyloidosis (ATTR) onsets due to the extracellular multiorgan deposition of misfolded transthyretin, a serum protein that synthesizes mainly in the liver. Two different forms of the disorder have been identified to date, namely wild type ATTR (wtATTR), previously referred to as "senile" since it was mainly diagnosed in the elderly; and an inherited ATTR (hAATR), caused by mutant transthyretin.

ATTR amyloidosis is often overlooked or misdiagnosed owing to its non-specific presentation.

Amyloid deposits can determine musculoskeletal manifestations, such as carpal tunnel syndrome (CTS), lumbar spinal canal stenosis (LSCS), or distal biceps tendon rupture (DBTR) several years before any cardiac manifestations, particularly in patients with wtATTR.

Cardiac manifestations of wtATTR (wtATTR-CA) include aortic stenosis, hypertrophic cardiomyopathy, heart failure with preserved ejection fraction, and hypertensive cardiomyopathy, although the cardiac signs and symptoms resemble those of other cardiovascular conditions of different etiology during the course of the disease [1].

We present radiological images of an 80-year-old man who had wtATTR-CA and LSCS. At the age of 65 , he had had bilateral CTS. Ten years later, he began to report pain and loss of strength in the lower limbs mainly localized in the buttocks and quadriceps. Computed tomography (CT) of the spine showed a LSCS due to ligamentum flavum hypertrophy (LFH) considered to result from fibrous degeneration (Figure 1A). ATTR-CA was diagnosed (Figure 1C) four years later. Genetic investigations yielded a negative result for hATTR. Upon further investigation, spinal magnetic resonance imaging (Figures 1D, E) and a second CT scan of the spine (Figure 1B) showed significant LFH with narrowing of the spinal canal.

The LF covers the rear surface of the spinal dural sac and has a protective effect on the spinal cord, controlling the extension of the intervertebral movement.

In the elderly, LFH is one of the most frequent causes of LSCS, and its onset has been related to a degenerative fibrotic condition. Patients usually complain of intermittent claudication, low back pain and leg numbness, and pain [2]. Several authors recently reported amyloid deposits in elderly patients with LFH and LSCS. In a recent study, where 250 patients underwent surgery for LSCS due to LFH, Eldhagen et al. identified an amyloid presence in $88.4 \%$ of the histological samples, and ATTR was found in $37 \%$ of the cases [3]. In a large group of 324 patients who underwent surgery for LSCS, Godara et al. found wtATTR in 13\% of the cases. wtATTR-CA was diagnosed in two of those cases. LF amyloid deposits could thus be considered an early manifestation of systemic ATTR disease [4]. Compared with CTS, LFH is a less known musculoskeletal wtATTR manifestation and is generally attributed to changes in fibrotic ligaments. Unfortunately, ${ }^{99 \mathrm{~m} T c-d i-}$ phosphanate scintigraphy, which is a useful diagnostic tool to detect wtATTR-CA, is not able to demonstrate LF amyloid deposits [5].

A correct interpretation of these manifestations, together with the cardiological 



Figure 1. A. Computed tomography (CT) of the spine showed a LSCS due to ligamentum flavum hypertrophy (yellow arrows). B. A second spinal CT after five years since the former revealed an increased thickness of the ligamentum flavum. C. ${ }^{99} \mathrm{mTc}$-3,3-diphosphono-1,2-propanodicarboxylic allows for a diagnosis of wtATTR. D, E. The magnetic resonance confirmed the ligamentum flavum hypertrophy

signs of the disease, allows for early diagnosis and faster access to therapy. For this reason, improved inter-specialty communication is required for managing patients with ATTR amyloidosis.

\section{Article information}

Conflict of interest: None declared.

Open access: This article is available in open access under Creative Common Attribution-Non-Commercial-No Derivatives 4.0 International (CC BY-NC-ND 4.0) license, allowing to download articles and share them with others as long as they credit the authors and the publisher, but without permission to change them in any way or use them commercially. For commercial use, please contact the journal office at kardiologiapolska@ptkardio.pl.

\section{REFERENCES}

1. Ruberg FL, Grogan M, Hanna M, et al. Transthyretin amyloid cardiomyopathy: JACC state-of-the-art review. J Am Coll Cardiol. 2019; 73(22): 28722891, doi: 10.1016/j.jacc.2019.04.003, indexed in Pubmed: 31171094.

2. Sun $C$, Zhang $H$, Wang $X$, et al. Ligamentum flavum fibrosis and hypertrophy: Molecular pathways, cellular mechanisms, and future directions. FASEB J. 2020; 34(8): 9854-9868, doi: 10.1096/f.202000635R, indexed in Pubmed: 32608536.

3. Eldhagen P, Berg S, Lund LH, et al. Transthyretin amyloid deposits in lumbar spinal stenosis and assessment of signs of systemic amyloidosis. J Intern Med. 2021; 289(6): 895-905, doi: 10.1111/joim.13222, indexed in Pubmed: 33274477.

4. Godara A, Riesenburger RI, Zhang DX, et al. Association between spinal stenosis and wild-type ATTR amyloidosis. Amyloid. 2021 [Epub ahead of print]: 1-8, doi: 10.1080/13506129.2021.1950681, indexed in Pubmed: 34263670.

5. Graziani A, Del Giudice E, Lisi M, et al. Scintigraphy showing the possible progression of transthyretin cardiac amyloidosis. Kardiol Pol. 2020; 78(3): 253-254, doi: 10.33963/KP.15125, indexed in Pubmed: 31917378. 